\title{
Quantum Capacitance of Aryldiazonium Modified Large Area Few- Layer Graphene Electrodes
}

Paula A. Brooksby, ${ }^{*},+$ Anna K. Farquhar, + Haidee M. Dykstra, $¥$ Mark R. Waterland, $\ddagger$ and Alison J. Downard $†$ +Department of Chemistry and MacDiarmid Institute for Advanced Materials and Nanotechnology, University of Canterbury, Private Bag 4800, Christchurch 8140, New Zealand

¥Chemistry - Institute of Fundamental Sciences and MacDiarmid Institute for Advanced Materials and Nanotechnology, Massey University, Private Bag 11 222, Palmerston North 4442, New Zealand

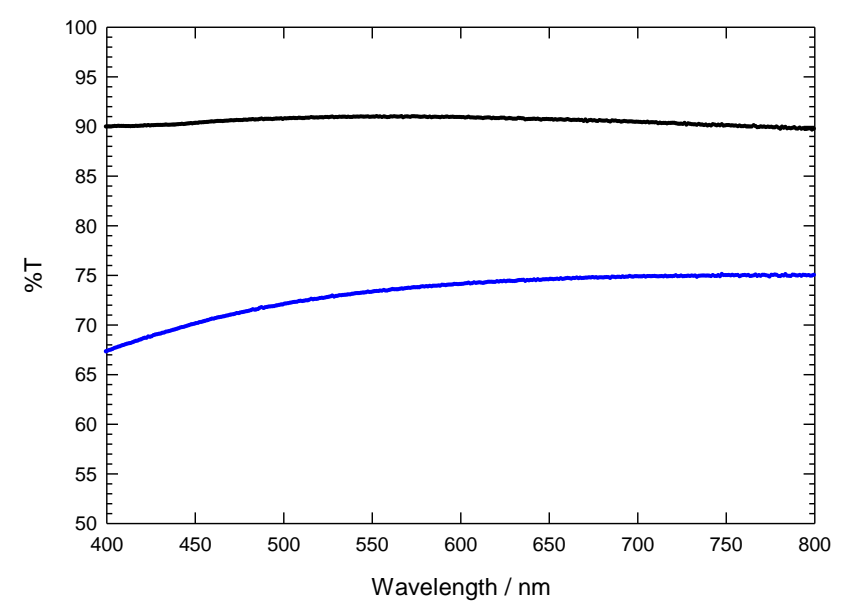

Figure S1. The UV spectra for (black) quartz and (blue) 7 min CVD growth of graphene growth of graphene. The \%T of a quartz slide was measured between 400 and $800 \mathrm{~nm}$ and then FLG sheets were mounted on top using the same water transfer procedure, and after drying the \% $\mathrm{T}$ was re-measured. The transparency change for each graphene layer is $2.3 \% \mathrm{~T}$. 
Quality of graphene. The Raman spectrum of FLG transferred onto a gold substrate is shown in Figure S2, with a curve fit of the $\mathrm{G}^{\prime}$ band requiring 8 peaks fixed at $24 \mathrm{~cm}^{-1} \mathrm{FWHM}$ as shown in Figure $\mathrm{S} 3$. When the number of layers is greater than 4 the Raman spectrum becomes difficult to curve fit and interpret with confidence. Hence, the UV data are the primary source for this information. However, the Raman spectra were used to observe the defect and disorder. The atmospheric pressure CVD growth method is expected to generate defective FLG and this is observed by the appearance of a D band (at $1345 \mathrm{~cm}^{-1}$ ). The ratio of the $D / G$ bands was $\sim 0.13$.

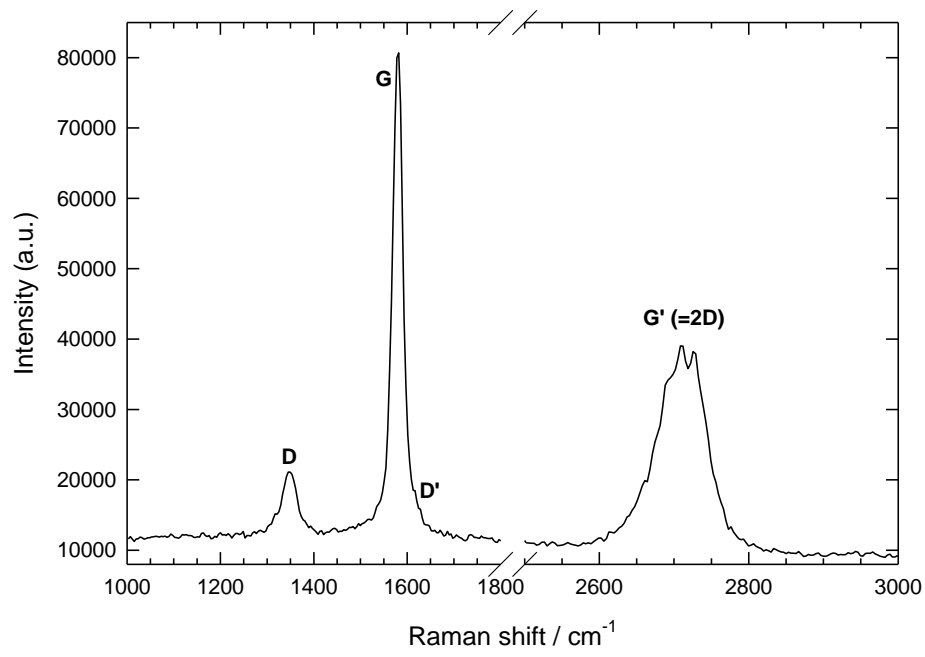

Figure S2. The Raman spectrum for FLG transferred onto a Au substrate.

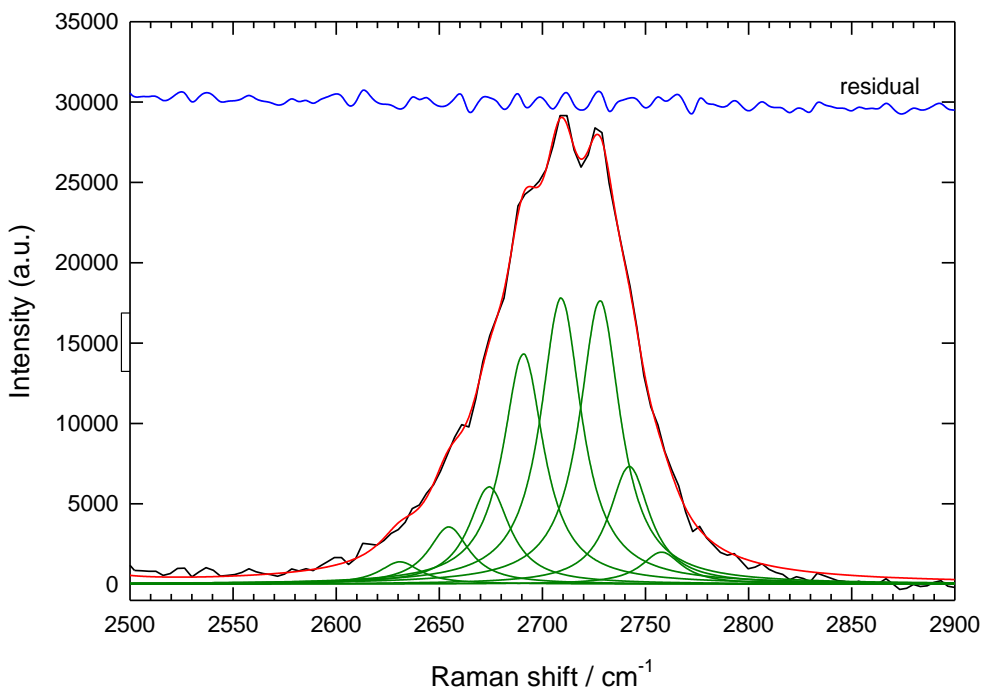

Figure S3. Curve fitting results for the $G^{\prime}$ (2D) band of graphene shown in Figure 2. (black) Measured spectrum, (red) curve fitted spectrum, (green) fitted peaks with fixed $24 \mathrm{~cm}^{-1} \mathrm{FWHM}$, and (blue) residual spectrum; offset and increased by a factor of 4 . 

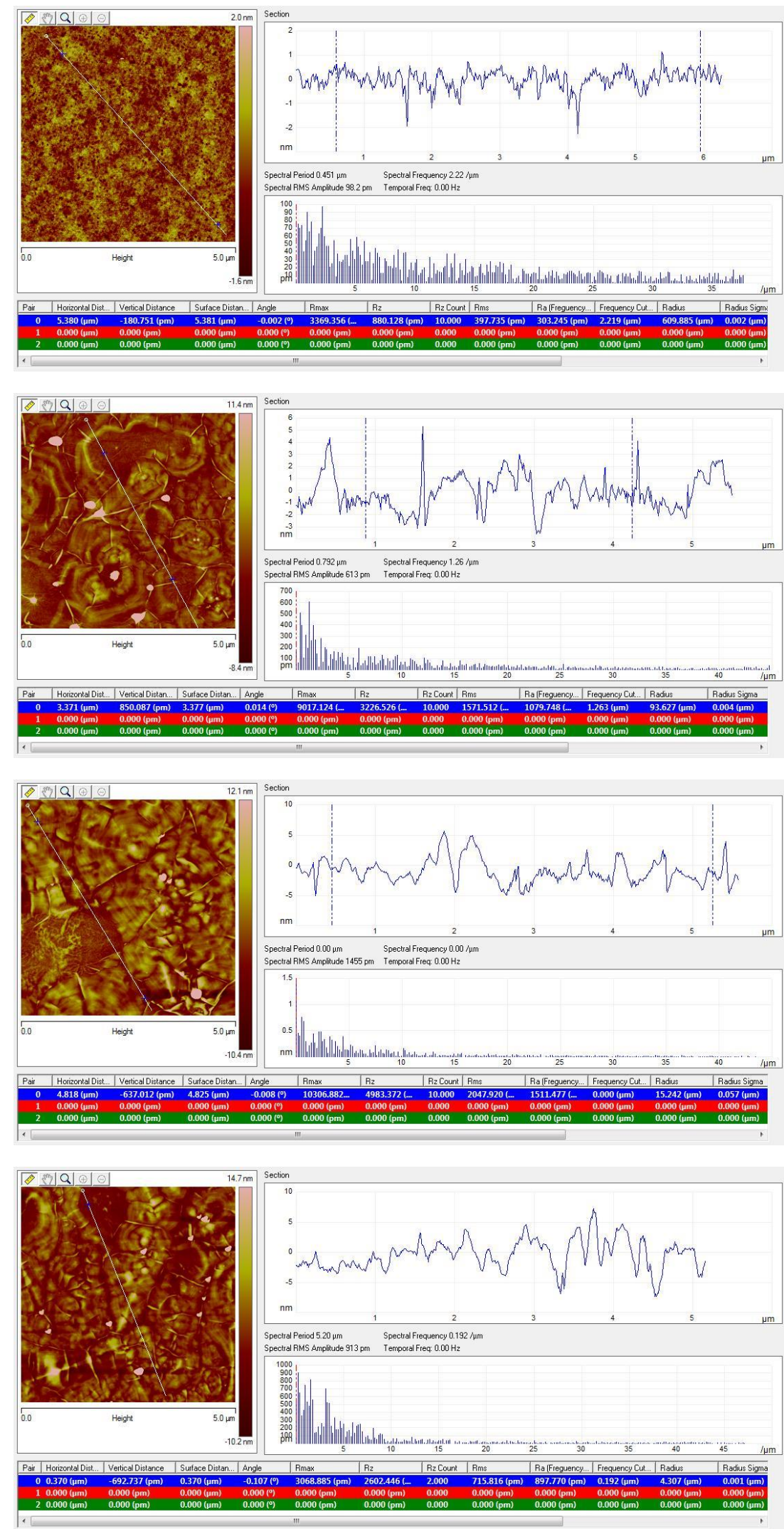

Figure S4. Line profiles from AFM images appearing in Figure 1 of text. (top to bottom) Epoxy resin, unmodified FLG, iodophenyl modified FLG and methoxypheny modified FLG on epoxy. 


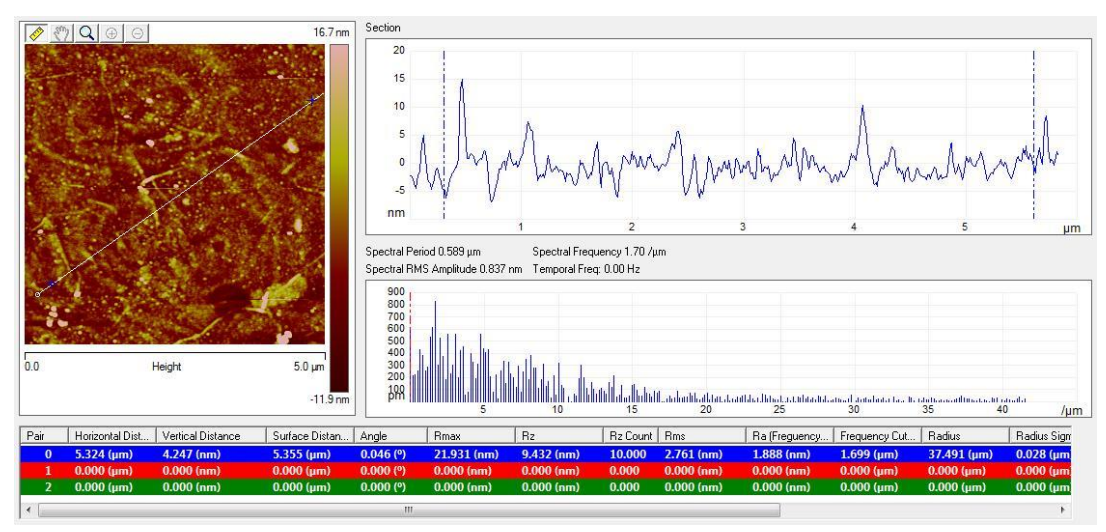

Figure S5: $\quad$ AFM image of a nitrophenyl modified FLG mounted on HOPG with the modifier groups uppermost. Shown is a 20 hour modification from $20 \mathrm{mM}$ diazonium salt solution.

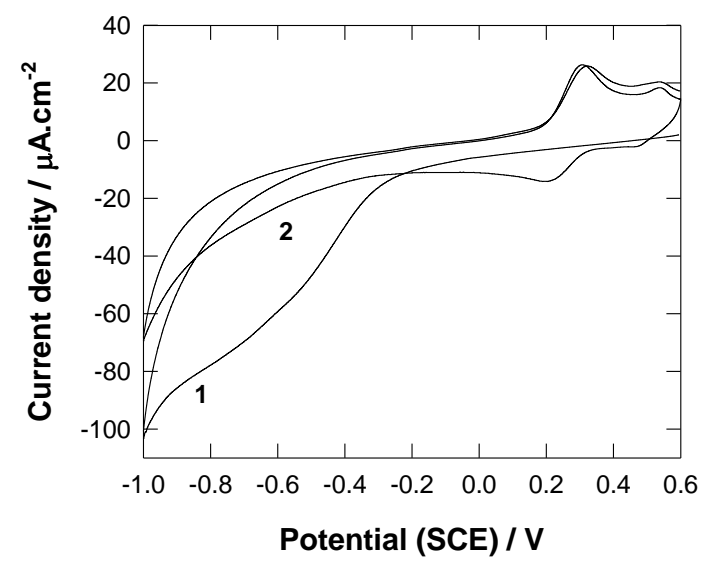

Figure S6: $\quad$ Electrochemistry of nitrobenzenediazonium modified FLG and the electrochemistry measured with the nitrophenyl groups sandwiched between graphene and the epoxy substrate. Shown is a 4 hour modification from a $7 \mathrm{mM}$ aryldiazonium salt solution.

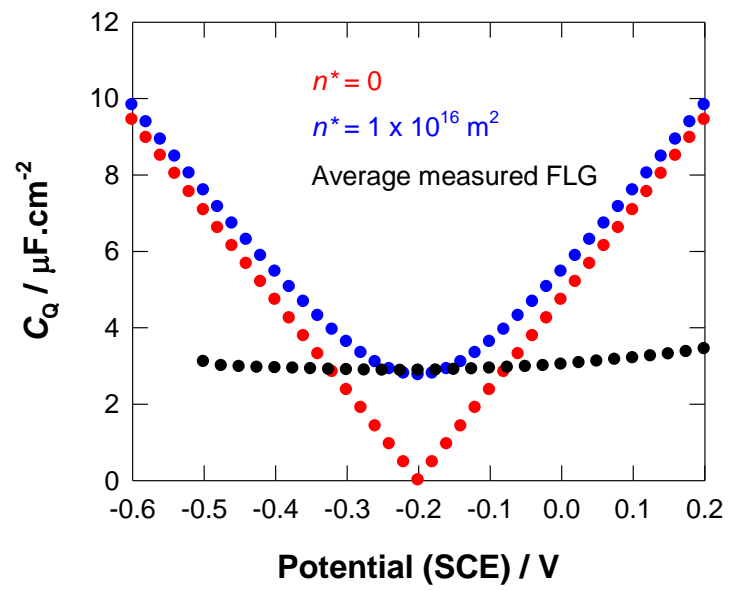

Figure S7: $\quad$ Graph showing the capacitance-voltage behaviour for pristine graphene taking into account the presence and absence of carriers, $n^{*}$, and experimentally measured FLG. The value $n *$ is either 0 or $1.0 \times$ $10^{16} \mathrm{~m}^{2}$. 